\title{
Detection of anomalies in NLO sulphamic acid single crystals by ultrasonic and thermal studies
}

\author{
GEORGE VARUGHESE* \\ Department of Physics, Catholicate College, Pathanamthitta, Kerala 689 645, India
}

MS received 17 October 2014; accepted 6 November 2015

\begin{abstract}
The ultrasonic pulse echo overlap technique (PEO) has been used to measure the velocities of $10 \mathrm{MHz}$ acoustic waves in sulphamic acid single crystals in the range of 300-400 K. This study evaluated all the elastic stiffness constants, compliance constants and Poisson's ratios of the crystal. The temperature variations of the elastic constants have been determined. The phase transition studies above room temperature were investigated using ultrasonic PEO technique. This study has suggested new weak elastic anomalies for the crystal around $330 \mathrm{~K}$. The transverse elastic constants $C_{44}$ and $C_{66}$ have shown clear thermal hysteresis of $2 \mathrm{~K}$. The present differential scanning calorimetric (DSC) studies carried out at a slow heating rate have also suggested weak phase transition around $331 \mathrm{~K}$. The present elastic and thermal studies have been substantiated by already reported DC electrical conductivity studies around $330 \mathrm{~K}$.
\end{abstract}

Keywords. Inorganic compounds; crystal growth; phase transitions; elastic properties; ultrasonic measurements.

\section{Introduction}

Sulphamic acid is a very interesting crystal with chemical formula $\mathrm{NH}_{3} \mathrm{SO}_{3}$, possesses orthorhombic symmetry exhibiting with possible piezo-electric and non-linear optical properties [1], X-ray diffraction (XRD) technique [2], infra-red [3], Raman [4], neutron diffraction scattering [5], thermal expansion studies and thermo-elastic properties [1] by resonance ultrasonic technique of Sulphamic acid were reported in the literature. X-ray diffraction study revealed that it crystallizes in the orthorhombic symmetry with space group Pbca. The X-ray, IR, Raman and neutron diffraction scattering studies stated that molecule exists in the crystal as zwitterions $\left(\mathrm{NH}_{3}^{+} \mathrm{SO}_{3}^{-}\right)$. The asymmetry of the quasi-tetrahedral $\left[\mathrm{NH}_{3} \mathrm{SO}_{3}\right]^{-}$ion suggests an unusually frequent formation of acentric species having polar properties like piezo-electric and non-linear optic effects. Since the hydrogen bonds are so disposed throughout the structure, they suggested that there exists no well-defined cleavage in any direction of the crystal. The thermo-elastic constant, $T_{\mathrm{ij}}$, thermal expansion coefficient, $\alpha_{\mathrm{ij}}$ and Gruneissen tensor, $G$ were reported in the literature [1] and they found that sulfamic acid possesses large value of $T_{\mathrm{ij}}$ since it has strong hydrogen bond. He observed thermal anomalies above $350 \mathrm{~K}$ for $\mathrm{CsNH}_{2} \mathrm{SO}_{3}$ and Betaine $\mathrm{NH}_{3} \mathrm{SO}_{3}$. Studies of Rapp [6] in differential scanning calorimetry (DSC) found that sulfamic acid exhibits a first order phase transition in the temperature range about $450 \mathrm{~K}$. Elastic constants are the only means to obtain information about the shear properties of the material. It provides information about the structural properties of the materials.

*Author for correspondence (gvushakoppara@yahoo.co.in)
Furthermore, elastic moduli are sensitive indicators of certain phase transformations and provide information about the interatomic forces. At a second order phase transition, while many thermodynamic quantities show no obvious evidence of the transition, elastic constants may show discontinuities, one can use the discontinuities to learn about the phase transition.

Aim of this study is to measure the elastic constants of sulphamic acid crystal using ultrasonic pulse echo overlap (PEO) technique. The elastic properties of sulphamic acid is well studied by measuring ultrasonic velocity in the crystal in certain specified crystallographic directions and evaluating the elastic stiffness constants, compliance constants and Poisson's ratios. The surface plots of phase velocity, slowness, Young's moduli and linear compressibility are made in $a-b, a-c$ and $b-c$ planes. Also, this study is intend to detect phase transitions in sulphamic acid single crystal above room temperature by temperature variation of second order elastic constants and DSC techniques. These results were substantiated with that of DC electrical conductivity studies reported in the literature [7] along different directions.

\section{Experimental}

\subsection{Sample preparation}

Large single crystals of sulphamic acid of size, $50 \times 35 \times$ $12 \mathrm{~mm}^{3}$, have been grown from supersaturated aqueous solution of the salt by slow evaporation technique over a period of 60-65 days. The temperature of the bath is maintained constant at $305 \mathrm{~K}$. The photograph of the grown crystal is depicted in figure 1 and the morphology of the crystal is 


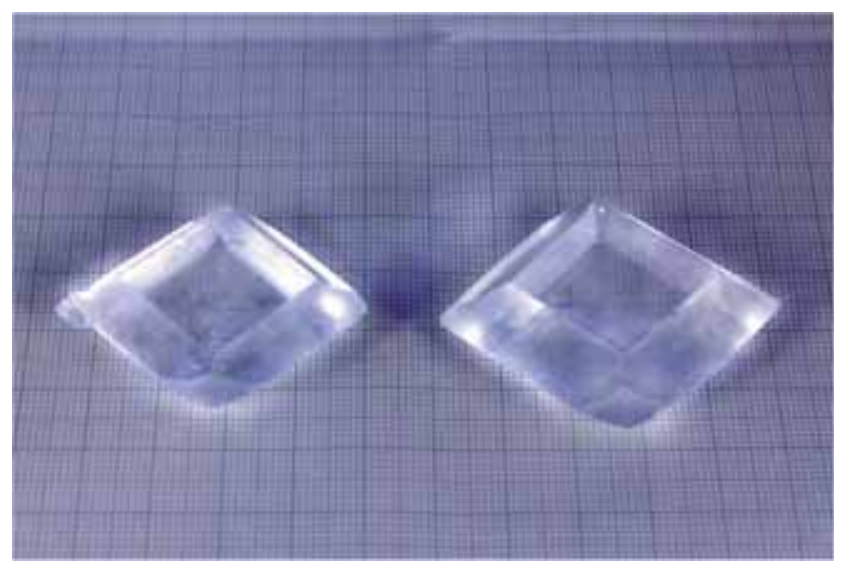

Figure 1. Photograph of grown sulphamic acid crystal.

shown in figure 2. The interfacial angles are measured using an accurate contact goniometer. The natural faces of the sample are identified by comparing the measured interfacial angles with that of the computed stereographic projection of the crystal planes [8] which are made using the lattice parameters $a=8.115 \AA, b=8.066 \AA$ and $c=9.255 \AA$. The good agreement between calculated and measured values of interfacial angles proves that our direction identification is correct. Bulk samples have been cut using a slow speed diamond wheel saw. Samples with a pair of parallel planes perpendicular to (100), (010), (001), (110), (011) and (101) directions have been prepared for ultrasonic wave velocity measurements. No cleavage is found in the crystal in any of the directions. The sample ends have been polished carefully using cerium oxide powder to optical reflection level. All cuttings are made very accurately; the error due to misorientation is below $\pm 0.5^{\circ}$.

\subsection{Ultrasonic velocity measurements}

The ultrasonic velocities are measured using the PEO technique. The details of measurement technique are explained [9]. A MATEC model 7700 pulse modulator and receiver system with its associated subunits have been used for the velocity measurements. Absolute velocities at room temperature (303 K) have been measured for the selected direction and modes. The McSkimin $\Delta t$ criterion [10] has been applied to correct the phase lag introduced by the bonding medium on the radio frequency wave (RF) echoes. The technique enables one to measure the absolute velocity to an accuracy of a few parts per million.

The temperature variation of the velocity of longitudinal and shear waves propagating along the various directions in the crystal (figures 5-9) have been determined in the range of $300-400 \mathrm{~K}$ by keeping the sample mounted on a suitable holder in a temperature-controlled chamber. Temperature variation measurements are carried out in temperature intervals of $1 \mathrm{~K}$ and in intervals of $0.1 \mathrm{~K}$ near transition points. Measurements have been carried out during cooling and heating cycles with the rate of temperature change in the

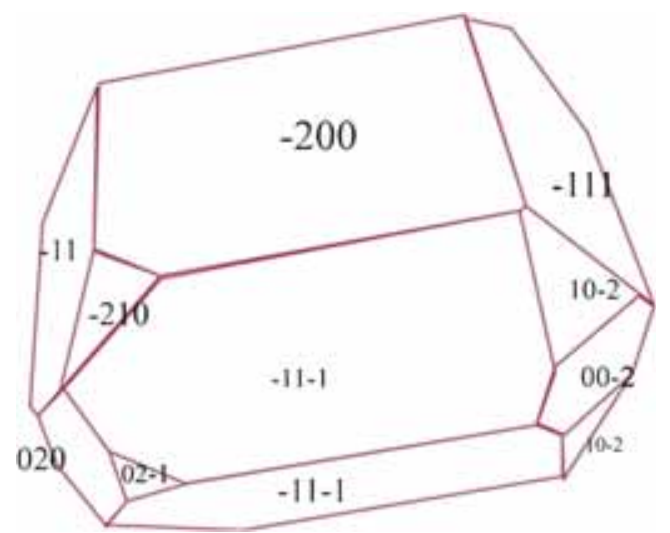

Figure 2. Morphology of sulphamic acid crystal.

range of $0.5-1 \mathrm{~K}$ per minute. The accuracy of the relative measurements of velocity is of the order of $10^{-5}$. We could not investigate the variation of velocity with temperature beyond $400 \mathrm{~K}$ because of bonding problems. The thermal expansion has been neglected, while measuring the variation of ultrasonic wave velocities with temperature.

\subsection{DC conductivity measurement}

Thin parallel plates of thickness $1 \mathrm{~mm}$ is cut from the crystal along (100), (010) and (001) planes using slow speed diamond wheel. The sample crystal is then placed in between two thin graphite plates which act as electrode. The DC conductivity was measured using electrometer (Keithley Electrometer model 2302) by applying a constant voltage of $10 \mathrm{~V}$. The temperature of the sample crystal was controlled by a programmable refrigerated circulator bath (JULABO GmbH FB50, Germany). The conductivity measurements were reported in the literature [7] in the temperature range of $300-430 \mathrm{~K}$. The temperature dependence of the ionic conductivity is usually given by the Arrhenius relation

$$
\sigma=\sigma_{0} \exp \left(-E_{\mathrm{g}} / K_{\mathrm{B}} T\right),
$$

where $E_{\mathrm{g}}$ is the activation energy, $K_{\mathrm{B}}$ the Boltzmann's constant and $\sigma$ the conductivity.

\subsection{Investigation of phase transition using DSC}

The thermal changes associated with this crystal by the DSC method based on ASTM E-537-98 using DSC-2920 was assessed. Thermal changes were observed in the range of $300-473 \mathrm{~K}$ at a very slow heating rate of $1^{\circ} \mathrm{min}^{-1}$. Annealed samples are used for this study. The peak areas in differential thermograms depend upon the mass of the sample, $m$, and enthalpy/unit mass is $\Delta H$ (energy associated with the transition). These variables are related by the equation

$$
A=-k G m \Delta H,
$$

where $A$ is the area of the peak, $G$ a calibration factor that depends on geometry and $k$ a constant related to the thermal conductivity of the sample. 


\section{Results and discussion}

\subsection{Measurements of elastic constants}

The elements of determinantal equation are defined by elastic constants and the direction cosines of the direction of propagation [8]. Non-zero elastic constants are shown in the determinant.

$$
C_{\mathrm{ij}}=\left|\begin{array}{cccccc}
C_{11} & C_{12} & C_{13} & 0 & 0 & 0 \\
C_{12} & C_{22} & C_{23} & 0 & 0 & 0 \\
C_{13} & C_{23} & C_{33} & 0 & 0 & 0 \\
0 & 0 & 0 & C_{44} & 0 & 0 \\
0 & 0 & 0 & 0 & C_{55} & 0 \\
0 & 0 & 0 & 0 & 0 & C_{66}
\end{array}\right| .
$$

$\mathrm{NH}_{3} \mathrm{SO}_{3}$ being an orthorhombic crystal, has the following nine second order elastic stiffness constants $C_{11}, C_{22}, C_{33}$, $C_{44}, C_{55}, C_{66}, C_{16}, C_{13}$ and $C_{23}$ (table 1). The diagonal elastic constants $C_{11}, C_{22}, C_{33}, C_{44}, C_{55}$ and $C_{66}$ have direct relationship with the suitable ultrasonic mode velocity given by

$$
C_{\mathrm{ij}}=\rho v^{2} .
$$

The relationships between elastic constants in relevant ultrasonic wave velocity for the orthorhombic system are reported in the literature [8]. The off-diagonal elastic constants can be found out by the following equation. The elastic constant $C_{12}$ can be calculated by measuring the velocity perpendicular to $a-b$ planes; here the angle is measured from $a$-axis, then

$$
\begin{aligned}
C_{12}=f_{\mathrm{ab}}= & \left\{\frac { 1 } { c ^ { 2 } s ^ { 2 } } \left[\left(s^{2} C_{11}+s^{2} C_{66}-\rho v^{2}\right)\right.\right. \\
& \left.\left.\times\left(c^{2} C_{66}+s^{2} C_{22}-\rho v^{2}\right)\right]\right\}^{1 / 2}-C_{66} .
\end{aligned}
$$

The elastic constant $C_{23}$ measured by propagating the sound with the velocity normal to the $b-c$ plane. The angle is measured from $b$-axis, then

$$
\begin{aligned}
C_{23}=f_{\mathrm{bc}}= & \left\{\frac { 1 } { c ^ { 2 } s ^ { 2 } } \left[\left(c^{2} C_{22}+s^{2} C_{44}-\rho v^{2}\right)\right.\right. \\
& \left.\left.\times\left(c^{2} C_{44}+s^{2} C_{33}-\rho v^{2}\right)\right]\right\}^{1 / 2}-C_{44} .
\end{aligned}
$$

The elastic constant $C_{13}$ can be measured by propagating the waves perpendicular to $a-c$ plane, where angle $\theta$ is measured from $c$-axis

$$
\begin{aligned}
C_{13}=f_{\mathrm{ac}}= & \left\{\frac { 1 } { c ^ { 2 } s ^ { 2 } } \left[\left(s^{2} C_{11}+c^{2} C_{55}-\rho v^{2}\right)\right.\right. \\
& \left.\left.\times\left(s^{2} C_{55}+c^{2} C_{33}-\rho v^{2}\right)\right]\right\}^{1 / 2}-C_{55},
\end{aligned}
$$

\begin{tabular}{|c|c|c|c|c|c|}
\hline Mode & $\begin{array}{l}\text { Direction of } \\
\text { propagation }\end{array}$ & $\begin{array}{l}\text { Direction of } \\
\text { polarization }\end{array}$ & $\begin{array}{l}\text { Velocity } \\
\text { measured } \\
V\left(\mathrm{~m} \mathrm{~s}^{-1}\right)\end{array}$ & $\begin{array}{c}\text { Elastic } \\
\text { constant } \\
C_{i j}(\mathrm{GPa})\end{array}$ & $\begin{array}{l}V-C_{i j} \\
\text { relationship }\end{array}$ \\
\hline $\mathrm{L}$ & $(100)$ & $(100)$ & $V_{1}=4157 \pm 4$ & $37.36 \pm 0.07$ & $C_{11}=\rho v_{1}^{2}$ \\
\hline $\mathrm{L}$ & (010) & (010) & $V_{2}=4541 \pm 4$ & $44.58 \pm 0.08$ & $C_{22}=\rho v_{2}^{2}$ \\
\hline $\mathrm{L}$ & $(001)$ & $(001)$ & $V_{3}=4218 \pm 4$ & $38.47 \pm 0.07$ & $C_{33}=\rho v_{3}^{2}$ \\
\hline $\mathrm{T}$ & (010) & $(001)$ & $V_{4}=2735 \pm 3$ & $16.17 \pm 0.03$ & $C_{44}=\rho v_{4}^{2}$ \\
\hline $\mathrm{T}$ & $(001)$ & (010) & $V_{5}=2748 \pm 3$ & - & $C_{44}=\rho v_{5}^{2}$ \\
\hline $\mathrm{T}$ & $(100)$ & $(001)$ & $V_{6}=2673 \pm 3$ & - & $C_{55}=\rho v_{6}^{2}$ \\
\hline $\mathrm{T}$ & (001) & (100) & $V_{7}=2726 \pm 3$ & $16.09 \pm 0.03$ & $C_{55}=\rho v_{7}^{2}$ \\
\hline $\mathrm{T}$ & (100) & (010) & $V_{8}=2843 \pm 3$ & $17.47 \pm 0.04$ & $C_{66}=\rho v_{8}^{2}$ \\
\hline $\mathrm{T}$ & $(010)$ & (100) & $V_{9}=2845 \pm 3$ & - & $C_{66}=\rho v_{9}^{2}$ \\
\hline QL & (110) & $c$ & $V_{10}=4408 \pm 4$ & $11.17 \pm 0.10$ & $C_{12}=f_{\mathrm{ab}}$ \\
\hline QL & (011) & $a$ & $V_{11}=4631 \pm 4$ & $19.61 \pm 0.15$ & $C_{23}=f_{\mathrm{bc}}$ \\
\hline QL & (101) & $b$ & $V_{12}=4525 \pm 4$ & $18.56 \pm 0.20$ & $C_{13}=f_{\mathrm{ac}}$ \\
\hline
\end{tabular}

where $s=\sin \theta, c=\cos \theta$ and $\theta$ is the angle of rotation with the respective axes. The angles are $44.83^{\circ}, 48.93^{\circ}$ and $41.25^{\circ}$ for $f_{\mathrm{ab}}, f_{\mathrm{bc}}$ and $f_{\mathrm{ac}}$ and are measured from the $a, b$ and $c$ axes, respectively. Then $v_{10}$ is the velocity of the wave in (110),

Table 1. Measured velocities and elastic constants of sulphamic acid crystal at $300 \mathrm{~K}$ along different directions with different polarizations. Also relation between elastic constants and velocity.

The abbreviation used have the following meaning: L, longitudinal; T, transverse. QL, quasi longitudinal; QT, quasi transverse; $s=\sin \theta, c=\cos \theta$ and $\theta$ is the angle of rotation with the respective axes. The angles are $44.83^{\circ}, 48.93^{\circ}$ and $41.25^{\circ}$ for $f_{\mathrm{ab}}, f_{\mathrm{bc}}$, and $f_{\mathrm{ac}}$, and are measured from the $a, b$ and $c$ axes, respectively. Then, $v$ is the velocity of propagation of respective mode and $\rho$ is the density of the sample $\left(=2.162 \mathrm{gm} \mathrm{cc}^{-1}\right.$ for sulphamic acid $)$. 
$v_{11}$ in the (011) and $v_{12}$ in the (101) direction and $\rho$ is the density of the sample ( $=2.162 \mathrm{~g} \mathrm{cc}^{-1}$ for sulphamic acid).

Of the propagation modes, 12 mode velocity measurements are sufficient to evaluate all the nine second-order elastic constants with cross checks possible on some of the values. Considering all experimental uncertainties, the absolute accuracy of elastic constant value is estimated to be better than $0.2 \%$ for diagonal elastic constant and $1 \%$ for off-diagonal elastic constants. In all the velocity measurements, the correct overlap identification and McSkimin $\Delta t$ criterion for bond correction have been applied using computer programme [11].

Some elastic stiffness constants of this crystal measured by this study (PEO method) shows appreciable deviation from that measured using resonant ultrasound technique. Out of the diagonal constants, the constants $C_{11}(5 \%), C_{33}(8.7 \%)$ and $C_{55}(9.8 \%)$ show deviation above $5 \%$, whereas constants $C_{22}(2.8 \%), C_{44}(2.1 \%)$ and $C_{66}(4 \%)$ exhibit deviation below $5 \%$. While off-diagonal constants $C_{12}(43 \%), C_{13}(19.8 \%)$ and $C_{23}(17.1 \%)$ have exhibited large deviations from the RUT values.

By measuring ultrasonic velocity in the sulphamic acid crystal in certain specified crystallographic directions, the anisotropy in the elastic properties of the crystal is studied. There are nine values of compliance constants which are the components obtained from the matrix inverse of elastic constants. Also there are six values for Poisson's ratios in orthorhombic crystal (table 2). The equations for Poisson's ratios are

$$
\begin{aligned}
& v_{21}=-\varepsilon_{22} / \varepsilon_{11}=-S_{21} / S_{11}, \\
& v_{31}=-\varepsilon_{33} / \varepsilon_{11}=-S_{31} / S_{11}, \\
& v_{12}=-\varepsilon_{11} / \varepsilon_{21}=-S_{12} / S_{22}, \\
& v_{32}=-\varepsilon_{33} / \varepsilon_{22}=-S_{23} / S_{22}, \\
& v_{23}=-\varepsilon_{221} / \varepsilon_{33}=-S_{23} / S_{33} .
\end{aligned}
$$

Table 2. Elastic stiffness constants, elastic compliance constants and Poisson's ratios of sulphamic acid crystal at $300 \mathrm{~K}$.

\begin{tabular}{lcl}
\hline $\begin{array}{l}\text { Elastic stiffness } \\
\text { constant (Gpa) }\end{array}$ & $\begin{array}{c}\text { Elastic compliance } \\
\text { constant } \\
\left(\times 10^{-10} \mathrm{~m}^{2} \mathrm{~N}^{-1}\right)\end{array}$ & Poisson's ratio \\
\hline$C_{11}=37.36 \pm 0.07$ & $S_{11}=0.353$ & $\nu_{12}=0.059$ \\
$C_{22}=44.58 \pm 0.08$ & $S_{22}=0.29$ & $\nu_{21}=0.048$ \\
$C_{33}=38.47 \pm 0.07$ & $S_{33}=0.409$ & $\nu_{23}=0.34$ \\
$C_{44}=16.17 \pm 0.03$ & $S_{44}=0.618$ & $\nu_{32}=0.479$ \\
$C_{55}=16.09 \pm 0.03$ & $S_{55}=0.622$ & $v_{13}=0.394$ \\
$C_{66}=17.47 \pm 0.04$ & $S_{66}=0.572$ & $\nu_{31}=0.456$ \\
$C_{12}=11.14 \pm 0.10$ & $S_{12}=-0.017$ & \\
$C_{23}=19.61 \pm 0.15$ & $S_{23}=-0.139$ & \\
$C_{13}=18.56 \pm 0.20$ & $S_{13}=-0.161$ & \\
\hline
\end{tabular}

\subsection{Polar plots of phase velocity, slowness, Young's modulus and linear compressibility}

With the aim of getting an insight into the anisotropy of elastic wave propagation in $\mathrm{NH}_{3} \mathrm{SO}_{3}$ single crystal, the velocity and slowness surface plots (figures 3 and 4) in the $a-b$ and $a-c$ planes, respectively, have been made following a well-known reported procedure [12]. The Young's modulus, $E$ in this direction of unit vector $n_{i}$ for an orthorhombic is given by

$$
\begin{aligned}
E= & {\left[S_{11} n_{1}^{4}+2 S_{12} n_{1}^{2} n_{2}+2 S_{13} n_{1}^{2} n_{3}^{2}+S_{22} n_{2}^{4}\right.} \\
& +2 S_{23} n_{2}^{2} n_{3}^{2}+S_{33} n_{3}^{4}+S_{44} n_{2}^{2} n_{3}^{2}+S_{55} n_{1}^{2} n_{3}^{2} \\
& \left.+S_{66} n_{1}^{2} n_{2}^{2}\right]^{-1} .
\end{aligned}
$$

The cross section of Young's moduli surfaces of $\mathrm{NH}_{3} \mathrm{SO}_{3}$ plotted in the $a-b, b-c$ and $a-c$ planes are shown in figure 5 .

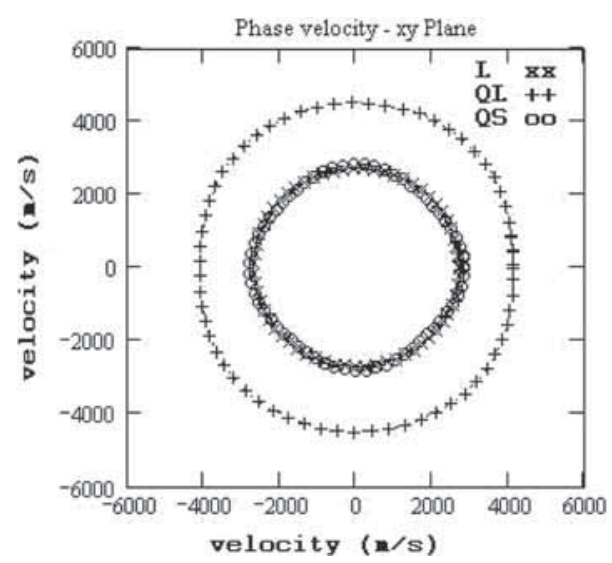

Figure 3. Surface plot of phase velocity in the xy plane of the crystal for longitudinal L, Quasi longitudinal QL and Quasi transverse QS.

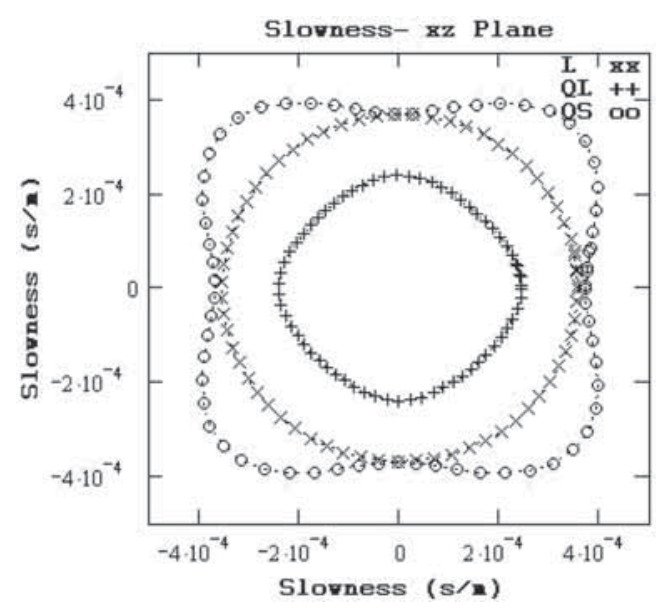

Figure 4. Surface plot of inverse of phase velocity (Slowness) in the xz plane of the crystal for longitudinal L, Quasi longitudinal QL and Quasi transverse QS. 
The linear compressibility [13] of an orthorhombic crystal in matrix form can be written as

$$
\begin{aligned}
\beta= & {\left[S_{11}+S_{12}+S_{13}\right] n_{1}^{2}+\left[S_{12}+S_{22}+S_{23}\right] n_{2}^{2} } \\
& +\left[S_{13}+S_{23}+S_{33}\right] n_{3}^{2} .
\end{aligned}
$$

The surface plots of linear compressibility of $\mathrm{HNH}_{2} \mathrm{SO}_{3}$ crystal in the $a-b, b-c$ and $a-c$ planes have been plotted (figure 6). The volume compressibility, $S_{i j k k}$ is an invariant parameter for a crystal. In matrix notation, it is given by

$$
S_{i i k k}=S_{11}+S_{22}+S_{33}+2\left[S_{12}+S_{23}+S_{13}\right],
$$

where $S_{\mathrm{ij}}$ 's are the corresponding compliance constants.

Bulk modulus of the crystal is

$$
K=1 / S_{i i k k} \text {. }
$$

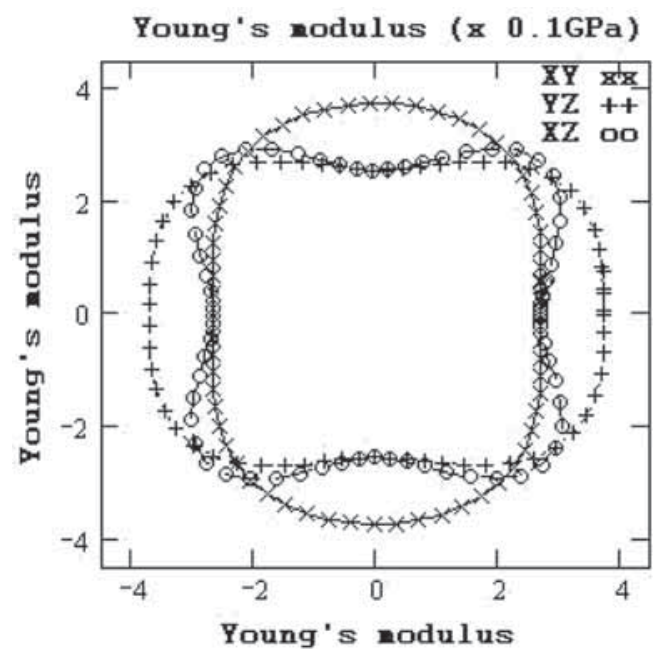

Figure 5. Surface plot of Young's modulus in the xy, yz, and xz planes of the crystal.

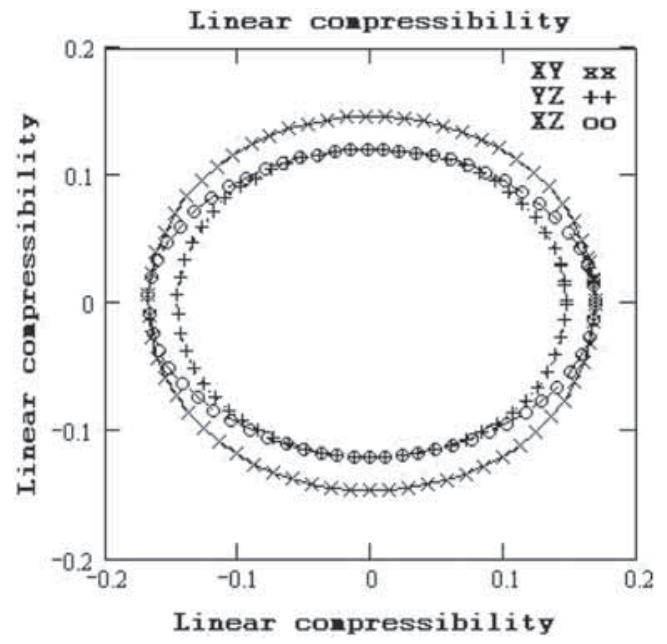

Figure 6. Surface plot of Linear compressibility in the xy, yz and $\mathrm{xz}$ planes of the crystal.
The volume compressibility of this crystal is evaluated as $0.437 \times 10^{-10} \mathrm{~N}^{-1} \mathrm{~m}^{-2}$ and bulk modulus is $22.88 \mathrm{GPa}$.

\subsection{Temperature variation of elastic stiffness constants}

The variations of the elastic constants with temperature in specified directions in the crystal are plotted in figures 7-11 in the range of 300-400 K by keeping the sample mounted on a suitable holder in a temperature-controlled chamber. The change in velocity with temperature with respect to the room temperature has been measured carefully by adjusting the $\mathrm{CW}$ oscillator frequency, keeping the selected RF echoes in the phase-matched condition. The accuracy of the relative measurements of velocity is of the order of $10^{-5}$. The rate of temperature change in all the measurements are in the range of $0.5-1 \mathrm{~K}$ per minute. We could not investigate the variation of velocity with temperature beyond $400 \mathrm{~K}$ because of bonding problems.

3.3a Anomalies around $330 \mathrm{~K}$ : Longitudinal elastic constants $C_{11}, C_{22}$ and transverse elastic constants $C_{44}, C_{55}$ and $C_{66}$ were subjected to temperature variation study. Of these,

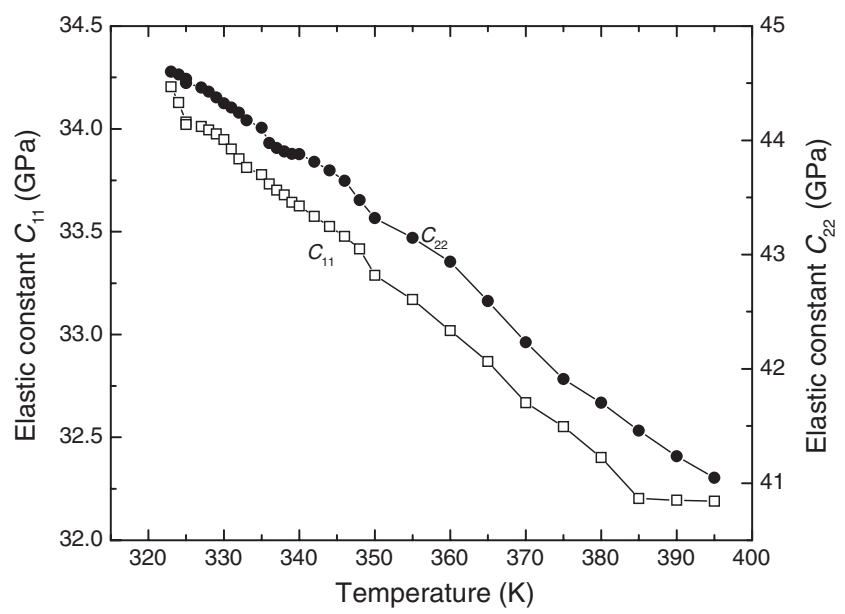

Figure 7. Variation of $C_{11}$ and $C_{22}$ with temperature.

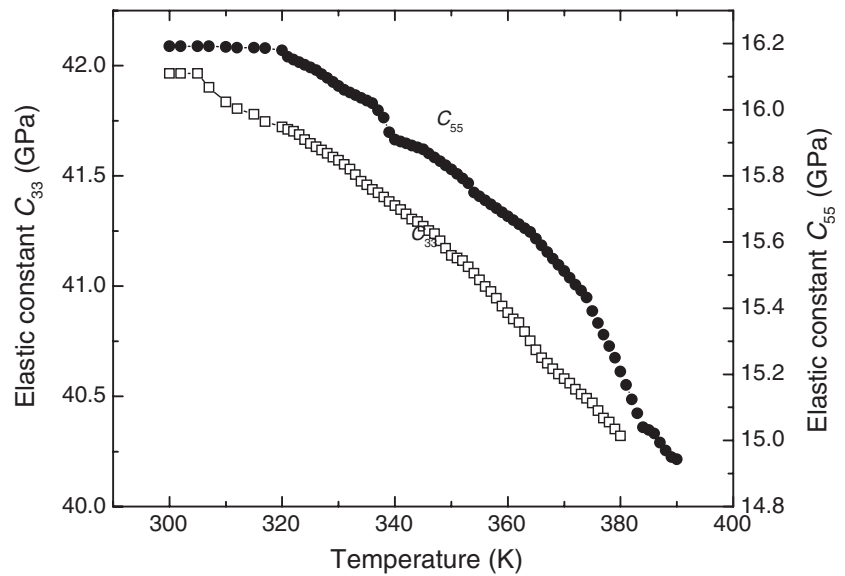

Figure 8. Variation of $C_{33}$ and $C_{55}$ with temperature. 
$C_{44}$ show a sharp decrease around 325 and $330 \mathrm{~K}$. The elastic constant $C_{11}$ also exhibited small anomalies around $330 \mathrm{~K}$. This means that shearing caused by tangential stress is considerably affected by thermal energy, since variation of shear elastic constants with thermal energy is appreciable. The constant $C_{33}$ has a general parabolic decrease as the

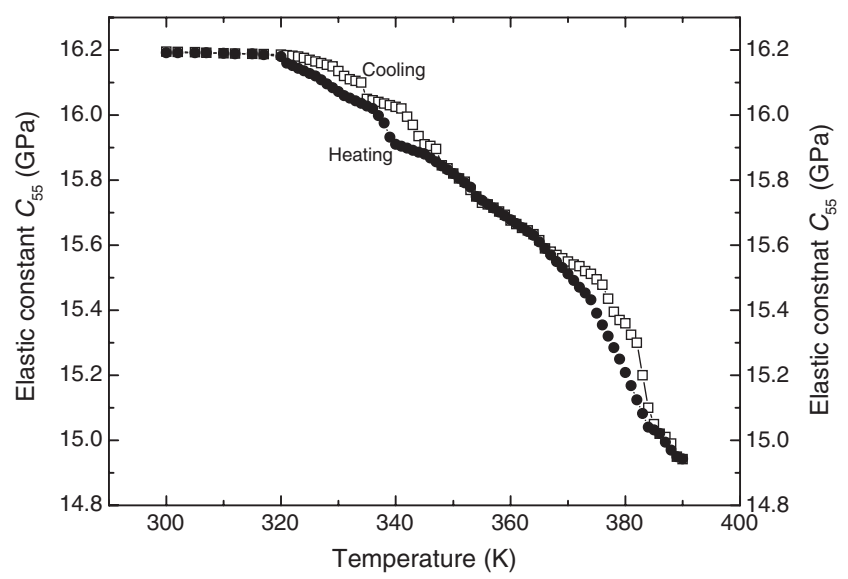

Figure 9. Variation of $C_{55}$ on heating and cooling cycles.

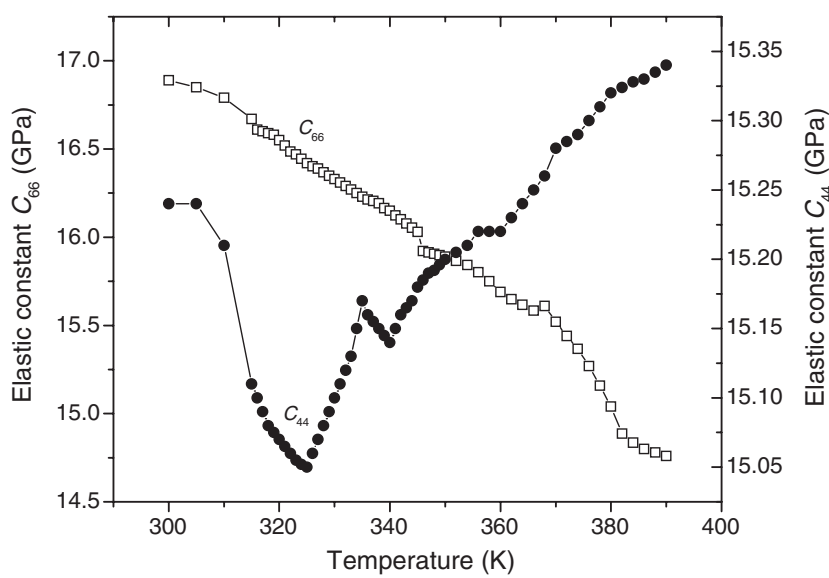

Figure 10. Variation of $C_{44}$ and $C_{66}$ with temperature.

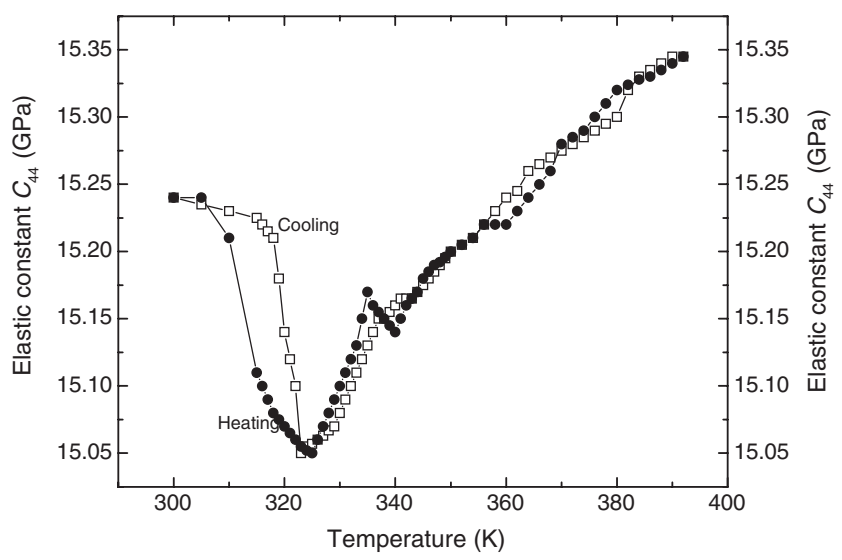

Figure 11. Variation of $C_{44}$ on heating and cooling cycles. temperature is increased. There is a clear thermal hysterisis of $2 \mathrm{~K}$ between cooling and heating cycles for this transition. During cooling, the transition took place at 327 and 333 K. During heating, it occurred at 325 and $331 \mathrm{~K}$. From the observed anomalies in the constants, we suggest a weak phase transition in the crystal around $331 \mathrm{~K}$.

3.3b Anomalies around $340 \mathrm{~K}:$ In the temperature variation study of elastic constants $C_{22}, C_{44}, C_{55}$ and $C_{66}$ have shown minor anomalies around $338 \mathrm{~K}$. The transverse elastic constants $C_{44}$ and $C_{55}$ have shown clear thermal hysteresis of $2 \mathrm{~K}$ between cooling and heating cycles for this transition.

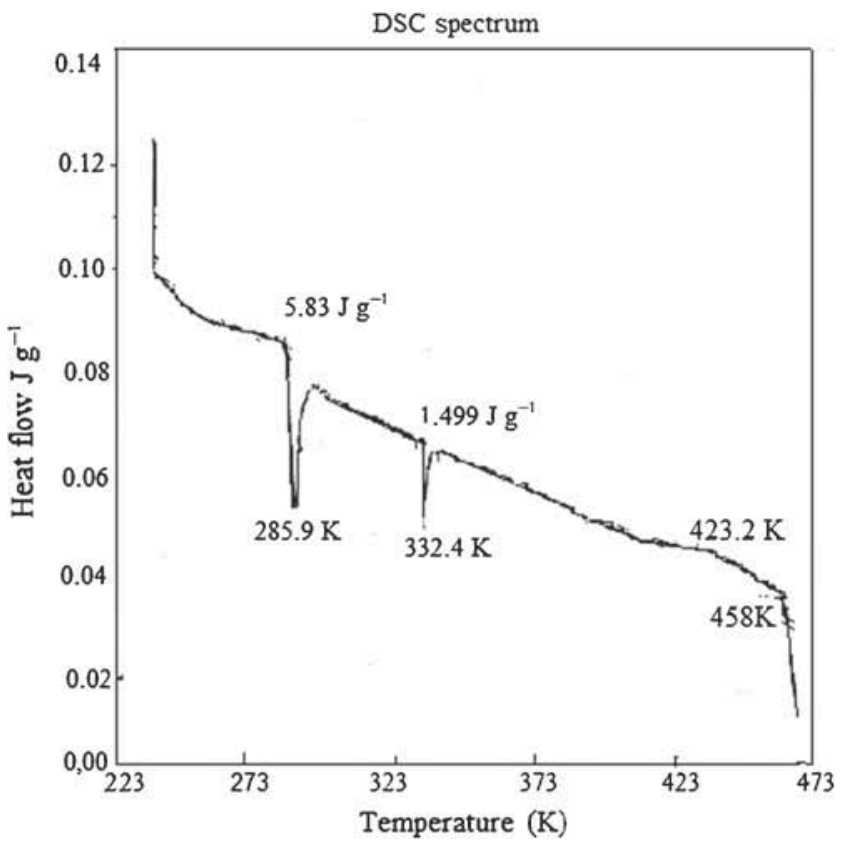

Figure 12. DSC scan of sulphamic acid.

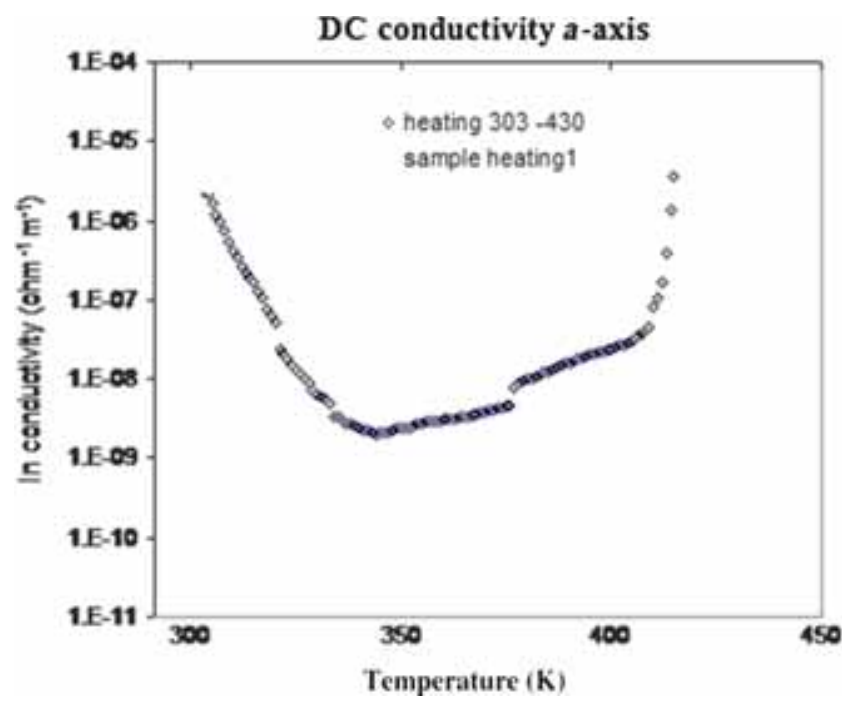

Figure 13. Variation of electrical conductivity with temperature along $a$-axis of the crystal in the temperature range $303-430 \mathrm{~K}$. 
Present measurements establish that on cooling, the transition occurs at $338 \mathrm{~K}$ and on heating, it takes place at $340 \mathrm{~K}$.

\subsection{DSC measurements}

3.4a Anomaly around $330 \mathrm{~K}$ : The present DSC study on sulphamic acid, in the temperature range of $238-473 \mathrm{~K}$, has been carried out at a very slow heating rate of $1^{\circ} \mathrm{min}^{-1}$. Annealed samples are used in this study. DSC spectrum (figure 12) shows a weak endothermic transitions centred around 285, 331 and pronounced transition around $458 \mathrm{~K}$. The endothermic energies involved in the transition are $5.83 \mathrm{~J} \mathrm{~g}^{-1}$ at $285.9 \mathrm{~K}$ and $1.499 \mathrm{~J} \mathrm{~g}^{-1}$ at $332.4 \mathrm{~K}$. Studies of Rapp [6] in DSC found that sulphamic acid exhibits a first order phase transition at the temperature of about $450 \mathrm{~K}$.

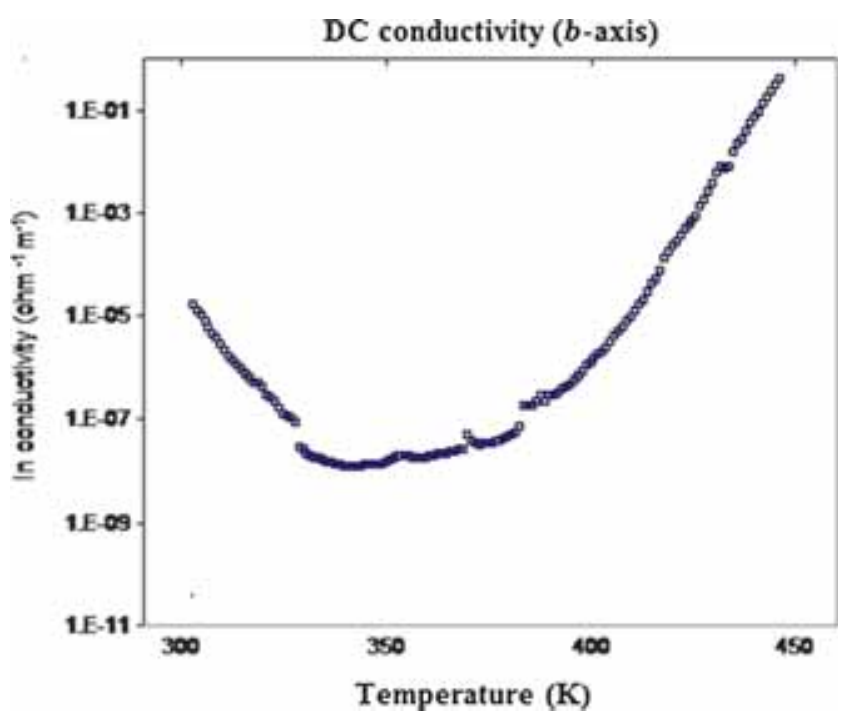

Figure 14. Variation of electrical conductivity with temperature along $b$-axis of the crystal in the temperature range $303-430 \mathrm{~K}$.

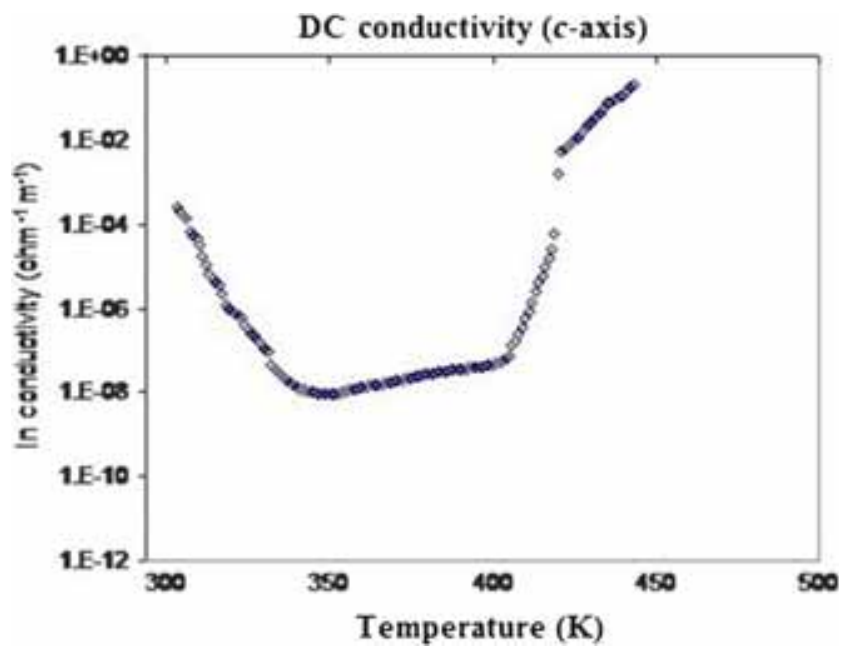

Figure 15. Variation of electrical conductivity with temperature along $c$-axis of the crystal in the temperature range $303-430 \mathrm{~K}$.
The thermal expansion has been neglected, while measuring the variation of ultrasonic wave velocities with temperature. The present DSC study do not show any anomalies at $340 \mathrm{~K}$. This may be due to the fact the weak anomaly at $340 \mathrm{~K}$ may not be associated with appreciable thermal change, whereas the sensitive ultrasonic technique can detect such a weak anomaly. In the thermal expansion studies [1], observed anomalies for the sulphamate family members, $\mathrm{CsNH}_{2} \mathrm{NO}_{3}$ and Betaine $\mathrm{NH}_{3} \mathrm{SO}_{3}$, are around $350 \mathrm{~K}$.

\subsection{DC conductivity measurements}

3.5a Anomaly around 330 and $340 \mathrm{~K}$ : The temperature dependence of the DC conductivity of the sulphamic acid single crystal along (100), (010) and (001) directions are shown in figures 13-15, respectively. From the reported figures, it has been found that the DC conductivity measurement [7] showed a large anomaly around $330 \mathrm{~K}$. As the temperature is increased from room temperature to $430 \mathrm{~K}$, the electrical conductivity decreases until around 340 and then, it increases very slowly up to $400 \mathrm{~K}$. After $400 \mathrm{~K}$, a drastic increase in the conductivity is noticed. The value of the conductivity is increased to the order of 4 at the temperature around $420 \mathrm{~K}$.

\section{Conclusions}

The temperature variation of elastic constants $C_{11}, C_{22}, C_{33}$, $C_{44}, C_{55}$ and $C_{66}$ in the temperature range of $300-400 \mathrm{~K}$ has been carried out. Of these, $C_{44}$ and $C_{55}$ have shown a sharp decrease around $330 \mathrm{~K}$. They have also exhibited weak anomalies around $340 \mathrm{~K}$. The thermal hysterisis associated with heating and cooling cycles for transition is about $2 \mathrm{~K}$. Elastic constants $C_{22}, C_{44}, C_{55}$ and $C_{66}$ have shown minor anomalies around $340 \mathrm{~K}$. The transverse elastic constants $C_{44}$ and $C_{66}$ have shown clear thermal hysterisis of $2 \mathrm{~K}$. The present DSC studies have also suggested weak phase transition around $332 \mathrm{~K}$ and do not show any anomalies around $340 \mathrm{~K}$. The DC conductivity measurement showed a large anomaly around $330 \mathrm{~K}$. In summary, from the observed anomalies in the elastic stiffness constants, DC conductivity and from the DSC investigations, we suggest a new weak phase transition in the crystal around $330 \mathrm{~K}$. More work is in progress about the nature of the new phase transition occurred in the crystal.

\section{Acknowledgement}

We thank M.G. University, Kottayam and CUSAT, Kochi, for technical support.

\section{References}

[1] Haussühl E and Haussühl S 1995 Z. Kristallogr. 210269

[2] Osaki K, Nitta I and Tadokoro H 1955 Bull. Chem. Soc. Jpn. 28524 
[3] Vaugnat A M and Wagner E L 1957 J. Chem. Phys. 2677

[4] Bats J W and Coppens P 1977 Acta Crystallogr. B 3337

[5] Sass R L 1960 Acta. Crystallalogr. 13320

[6] Rapp K W 1992 Kristallographie einiger Amidosulfate ein-und zweiwertiger kationen (Munchen: Diplomarbeit Universitat).

[7] Kumar A S, Louis G and Varghese G 2010 Cryst. Res. Technol. 458879
[8] Godfrey L and Philip J 1994 J. Appl. Phys. 752393

[9] Papadakis E P 1976 in Physical acoustics (eds) W P Mason and R N Thursto (New York: Academic Press) vol. XII

[10] McSkimin H J and Andreatch P 1962 Acoust. Soc. Am. 34609

[11] Godfrey L and Philip J 1995 Acoust. Lett. 1911

[12] Alex A V and Philip J 2000 Mater. Sci. Eng. B 90241

[13] Musgrave M J P 1970 Crystal acoustics: introduction to study of elastic waves and vibration in crystals (Holden-Day) 